
AIDS
DOI: $10.1097 / Q A D .0000000000001197$

\section{Life expectancy trends in adults on antiretroviral treatment in South Africa}

Running head: Life expectancy trends in ART patients

Leigh F. JOHNSON ${ }^{1}$

Olivia KEISER ${ }^{2}$

Matthew P. FOX ${ }^{3,4}$

Frank TANSER ${ }^{5}$

Morna CORNELL ${ }^{1}$

Chris J. HOFFMANN ${ }^{6,7}$

Hans PROZESKY ${ }^{8}$

Andrew BOULLE ${ }^{1}$

Mary-Ann DAVIES ${ }^{1}$

For the International Epidemiologic Databases to Evaluate AIDS Southern Africa (IeDEASA) collaboration

1. Centre for Infectious Disease Epidemiology and Research, University of Cape Town, South Africa

2. Institute of Social and Preventive Medicine, University of Bern, Bern, Switzerland

3. Departments of Epidemiology and Global Health and Development, Boston University, USA

4. Health Economics and Epidemiology Research Office, Department of Medicine, Faculty of Health Sciences, University of the Witwatersrand, South Africa

Copyright @ 2016 Wolters Kluwer Health, Inc. Unauthorized reproduction of this article is prohibited. 
5. Africa Centre for Health and Population Studies, University of KwaZulu-Natal, South Africa

6. Aurum Institute, South Africa

7. Division of Infectious Diseases, Johns Hopkins School of Medicine, USA

8. Division of Infectious Diseases, Department of Medicine, University of Stellenbosch and Tygerberg Academic Hospital, South Africa

Correspondence to:

Dr Leigh Johnson

Centre for Infectious Disease Epidemiology and Research

Faculty of Health Sciences

University of Cape Town

Anzio Road

Observatory 7925

Cape Town

South Africa

Tel: +27214066981

Fax: +27 214066764

Email: Leigh.Johnson@uct.ac.za

Word count (excluding cover sheet, abstract, tables and references): 1799

Conflicts of interest and sources of funding: The authors declare no conflicts of interest. This research was funded by the National Institutes of Health (grant U01AI069924).

Copyright @ 2016 Wolters Kluwer Health, Inc. Unauthorized reproduction of this article is prohibited. 
Abstract

Background: Previous studies have reported improvements in life expectancies of patients on antiretroviral treatment (ART) over time, but it is not clear whether these improvements are explained by changes in baseline clinical characteristics, longer duration on ART, or changes in clinical practices.

Method: Two parametric survival models were fitted to mortality data from South African ART cohorts that had linked patient records to the national vital registration system. The first model estimated mortality by age, sex, cohort, baseline CD4 count, time since ART initiation and period of ART initiation; the second model included only age, sex, cohort and period of follow-up. Life expectancies were calculated from the estimated mortality rates.

Results: The first model estimated little change in mortality over time: women starting ART at age 35, at CD4 counts of 200 cells/ $\mu$ or higher, had life expectancies of 32.7 (95\% CI: 31.6-33.6), 32.4 (95\% CI: 31.3-33.4) and 33.0 (95\% CI: 32.0-34.1) in the 2001-2006, 20072009 and 2010-2014 periods respectively. However, the second model estimated a significant improvement in life expectancy; for all women on ART at age 35, corresponding life expectancies were 13.0 (95\% CI: 12.1-14.2), 20.4 (95\% CI: 19.5-21.4) and 26.1 (95\% CI: 25.2-26.9) respectively.

Conclusions: Although life expectancies in South African ART patients have improved over time, these improvements are not observed after controlling for changes in baseline CD4 count and ART duration. This suggests that changes in clinical practice and programme scale have had little impact on ART mortality in South Africa.

Key words: HIV; AIDS; life expectancy; highly active antiretroviral therapy; South Africa 


\section{Introduction}

Several recent studies have shown improving life expectancies over time, among adults receiving ART [1-4]. However, it is not yet clear to what extent this improvement is due to improvements in patient care, changes in baseline clinical characteristics and/or changes in average ART durations [5]. Because most previously-published analyses of life expectancy trends do not simultaneously control for changes over time in baseline CD4 distributions and changes in ART durations, the true extent to which clinical changes are leading to improvements in mortality is uncertain.

In a previous analysis of life expectancies of adults starting ART in South Africa, we showed that life expectancies at the point of ART initiation were approximately $80 \%$ of those in HIVnegative adults of the same age, if ART was initiated at CD4 counts $>200$ cells $/ \mu 1$ [6]. However, this analysis was limited by lack of long-term follow-up data and the relatively small numbers of patients starting ART at higher CD4 counts. Life expectancy forecasts are sensitive to mortality estimates at longer ART durations, and it is therefore important to update these forecasts as more data become available on patients with longer follow-up durations.

South Africa is in a unique position within Africa, being the only country with both a large HIV burden and a well-functioning death notification system, which is estimated to record around $95 \%$ of all adult deaths [7]. This makes it possible to obtain reasonably accurate estimates of mortality, which are not biased by the high loss to follow-up observed in many ART cohorts in developing countries [8]. This paper aims to produce updated estimates of life expectancies in South Africa and to test whether life expectancies of ART patients have improved after controlling for changes in baseline CD4 and ART duration. 


\section{Methods}

This analysis is based on eight South African cohorts providing ART to adults (listed in Table S1). All cohorts obtained ethical approval from relevant local institutions before contributing anonymized data to the collaborative analysis. In addition, the collaboration received ethical approval from the University of Cape Town Human Research Ethics Committee to receive and analyse these data. Each cohort linked patient records to the national vital registration system, for all patients who had a national identity number (ID), to determine their vital status (and date of death where relevant).

Patients were included in the analysis if they were aged 15 or older at ART initiation and started ART in 2001 or later. Patients were excluded if they had a missing baseline CD4 count or a viral load of $<400$ copies $/ \mathrm{ml}$ prior to ART initiation. The analysis was limited to patients with recorded IDs; this restriction was introduced because of concern that some cohorts may have incorporated data from the vital registration system into patient records, which would invalidate the inverse probability weighting approach that was previously applied to account for differences in the recording of deaths between patients with and without IDs [6]. Patients with recorded IDs have previously been shown to be similar to patients without recorded IDs in their clinical and demographic characteristics, although the capturing of patient ID information has become more frequent in recent years [9].

Patients were considered dead if death was recorded on either the patient record system or the vital registration system (in the event of discrepancies in the recorded date of death, the date on the patient record was assumed to be correct). Observation time was calculated from the date when the patient first started ART. Observation was censored at the recorded date of 
death or 30 days prior to the date on which the patient's record was linked to the vital registration system, whichever date occurred first (the 30-day offset was included to reduce bias due to possible late registration of deaths). No censoring was applied if the patient interrupted ART, transferred to other ART services or did not return to the clinic, since the only outcome of interest was death after ART initiation, which could be determined from the vital registration system.

The statistical approach to estimating life expectancy is the same as that described previously [6]. Briefly, two parametric relative survival models [10] were fitted to the mortality data to identify predictors of mortality in excess of that expected in HIV-negative South Africans [11]. In the first model, explanatory variables included cohort, age, baseline CD4 count (categorized as shown in Table S1), ART duration and period of ART initiation (three periods were defined, with cut-offs at the end of 2006 and 2009 to ensure similar proportions of patients in each period). In the second model, to be consistent with the approach adopted in other analyses of life expectancy trends [1-4], explanatory variables included cohort, age and period of ART follow-up (defined by the same cut-offs). The relative survival models were fitted separately for males and females, and (in the first model) for periods $\leq 12$ months and $>$ 12 months after ART initiation. Parametric bootstrap sampling was used to calculate $95 \%$ confidence intervals. For each bootstrap sample, tables of age-specific mortality rates were calculated (for each integer ART duration in the case of the first model), and from these mortality tables, life expectancies were calculated for all age, sex, cohort, CD4 and period categories. Life expectancies were averaged across the eight cohorts and across 1000 bootstrap samples. Statistical analyses were performed in STATA 13.0 (StataCorp) and C++. 
Sensitivity analyses were performed to assess the effect of including patients with missing baseline CD4 values, assigning CD4 values using multiple imputation [12]. Sensitivity analyses also assessed Cox proportional hazard models.

\section{Results}

From an initial database of 106498 patients, 37383 (35\%) were excluded due to absence of IDs, $13542(13 \%)$ were excluded due to missing baseline CD4 measures and 3084 (3\%) were excluded due to baseline viral load levels below 400 copies/ml. Analyses were performed on the remaining 52489 patients. Baseline characteristics are summarized in Table S1: http://links.Iww.com/QAD/A950 29.1\% of patients started ART in the 2001-2006 period, $42.4 \%$ started ART in the 2007-2009 period, and the remaining $28.5 \%$ started ART in 2010 or later. Patients were followed for a total of 204508 person years (average follow-up duration of 3.90 years). A total of 8376 deaths occurred, yielding a crude mortality rate of 41.0 per 1000 person years.

The coefficients of the first relative survival model are summarized in Supplementary Table S2. http://links.Iww.com/QAD/A950 After adjusting for differences in baseline covariates and differences in treatment durations, HIV-related mortality rates in patients starting ART before 2007 were similar to those in patients starting ART in the 2007-2009 and 2010-2014 periods. Men starting ART at age 35, at CD4 counts of 200 cells/ $\mu 1$ or higher, had life expectancies of 26.1 years (95\% CI: 24.9-27.3), 26.4 years (95\% CI: 25.2-27.5) and 26.7 years (95\% CI: $25.3-28.0)$ in the $2001-2006,2007-2009$ and $2010-2014$ periods respectively (80-82\% of HIV-negative life expectancy). The corresponding life expectancies for women were 32.7 years (95\% CI: 31.6-33.6), 32.4 years (31.3-33.4) and 33.0 years (95\% CI: 32.0-34.1) 
respectively (82-84\% of HIV-negative life expectancy). Similarly, no trends in life expectancy were apparent when considering other baseline CD4 categories and baseline ages (Figure 1).

In the second survival model, which did not control for ART duration or baseline CD4 count, HIV-related mortality rates declined significantly in successive follow-up periods (Table S3, http://links.Iww.com/QAD/A950). As a result, life expectancies increased over time (Figure 2). For men on ART at age 35, life expectancies increased from 9.9 (95\% CI: 9.2-10.6) in 20012006 to 15.9 (95\% CI: $15.3-16.6)$ in $2007-2010$ and 21.1 (95\% CI: 20.3-21.8) in 2010-2014. Corresponding estimates for women aged 35 were 13.0 (95\% CI: 12.1-14.2), 20.4 (95\% CI: 19.5-21.4) and 26.1 (95\% CI: 25.2-26.9) respectively.

Results from the first model were similar when patients with missing baseline CD4 values were included in the analysis, with imputation of missing CD4 values (Supplementary Table S4, http://links.Iww.com/QAD/A950). No trend in mortality was observed when fitting Cox proportional hazards models that more accurately controlled for baseline CD4 count and ART duration (Supplementary Table S5, http://links.Iww.com/QAD/A950).

\section{Discussion}

This analysis shows that life expectancy estimates are sensitive to the calculation method used, and care is therefore required in the interpretation of life expectancy statistics. The first model yields a prognostic measure for a patient starting ART with a given CD4 count, while the second model is a summary indicator of mortality among all patients on ART in a particular calendar period. Consistent with other studies that have used the second model [1- 
4], our analysis shows an improvement in aggregate mortality levels in ART patients. However, after controlling for changes over time in baseline CD4 count and ART duration, the second model suggests no improvement in life expectancy. The lack of improvement might be considered surprising in view of various improvements in clinical practices. These include the change in South African ART guidelines in 2010 to recommend the use of tenofovir instead of stavudine in first-line drug regimens [13]; the introduction of fixed dose combination ART in 2013, which has been shown to be associated with improved adherence and virological suppression [14]; and the introduction of adherence clubs since 2011 in some centres [15]. It is possible that these gains may have been offset by increases in loss to follow-up [16], and possible reductions in quality of care as programmes have expanded in size.

The results from the first model reaffirm our previous findings of near-normal life expectancies in South African patients starting ART at CD4 counts above 200 cells/ $\mu 1$ [6]. This analysis improves on our previous analysis by including a broader range of ART cohorts (two additional cohorts added to the six cohorts considered previously), a greater number of patients (52 489 patients compared to 37740 previously) and more than double the average length of observation (3.90 years compared to 1.84 years previously).

This study is limited by a lack of reliable data on the vital status of patients without IDs. Most ID information is missing because it was not requested, but a substantial proportion of those without ID information are individuals who do not have South African IDs, many of them foreign nationals. Although previous studies have shown that patients with recorded IDs are similar to those without in terms of demographic and clinical characteristics [9], we cannot exclude the possibility that patients without IDs may have different mortality levels. Another 
limitation is that the IeDEA cohorts might not be typical of the South African ART programme generally, particularly as most cohorts are based in urban areas. The generalizability of these findings to other settings within South Africa is therefore unclear.

In summary, previously-observed improvements if life expectancies of patients on ART, over time, might be explained mostly by changes in baseline CD4 count and ART duration. In South Africa there is little evidence to suggest that life expectancy improvements are attributable to changes in clinical practice.

\section{Acknowledgements}

We thank the IeDEA-WHO Collaboration for their guidance and expertise. This research was funded by the National Institutes of Health (grant U01AI069924).

$\mathrm{LJ}, \mathrm{AB}$ and MD conceived the study. MF, FT, $\mathrm{CH}, \mathrm{HP}$ and $\mathrm{AB}$ contributed to data collection. $\mathrm{LJ}, \mathrm{OK}$ and MC contributed to the data analysis. LJ drafted the manuscript with input from all authors. All authors have read and approved the final manuscript.

\section{Supplementary digital content}

All supplemental digital content is contained in the text file SupplementaryMaterial2.doc. 


\section{References}

1. Nsanzimana S, Remera E, Kanters S, Chan K, Forrest JI, Ford N, et al. Life expectancy among HIV-positive patients in Rwanda: a retrospective observational cohort study. Lancet Global Health 2015; 3:e169-177.

2. Hogg R, Lima V, Sterne JAC, Grabar S, Battegay M, Bonarek M, et al. Life expectancy of individuals on combination antiretroviral therapy in high-income countries: a collaborative analysis of 14 cohort studies. Lancet 2008; 372:293-299.

3. May M, Gompels M, Delpech V, Porter K, Post F, Johnson M, et al. Impact of late diagnosis and treatment on life expectancy in people with HIV-1: UK Collaborative HIV Cohort (UK CHIC) Study. British Medical Journal 2011; 343:d6016.

4. Samji H, Cescon A, Hogg RS, Modur SP, Althoff KN, Buchacz K, et al. Closing the gap: increases in life expectancy among treated HIV-positive individuals in the United States and Canada. PLoS One 2013; 8:e81355.

5. Egger M, Johnson LF. Estimating trends in life expectancy in HIV-positive individuals. Lancet Global Health 2015; 3:e122-123.

6. Johnson LF, Mossong J, Dorrington RE, Schomaker M, Hoffmann CJ, Keiser O, et al. Life expectancies of South African adults starting antiretroviral treatment: Collaborative analysis of cohort studies. PLoS Medicine 2013; 10:e1001418.

7. Johnson LF, Dorrington RE, Laubscher R, Hoffmann CJ, Wood R, Fox MP, et al. A comparison of death recording by health centres and civil registration in South Africans receiving antiretroviral treatment. Journal of the International AIDS Society 2015; 18:20628. 
8. Fox MP, Rosen S. Retention of adult patients on antiretroviral therapy in low- and middle-income countries: systematic review and meta-analysis 2008-2013. Journal of Acquired Immune Deficiency Syndromes 2015; 69:98-108.

9. Boulle A, Schomaker M, May MT, Hogg RS, Shepherd BE, Monge S, et al. Mortality in patients with HIV-1 infection starting antiretroviral therapy in South Africa, Europe, or North America: a collaborative analysis of prospective studies. PLoS Medicine 2014; 11:e1001718.

10. Dickman PW, Sloggett A, Hills M, Hakulinen T. Regression models for relative survival. Statistics in Medicine 2004; 23:51-64.

11. Actuarial Society of South Africa. ASSA2008 AIDS and Demographic Model. 2011. Available: http://aids.actuarialsociety.org.za. Accessed 5 April 2011

12. Molenberghs G, Kenward MG. Missing Data in Clinical Studies. Chichester, United Kingdom: John Wiley \& Sons; 2007.

13. Department of Health. Clinical guidelines for the management of HIV and AIDS in adults and adolescents. 2010. Available: http://www.doh.gov.za/docs/facts-f.html. Accessed 30 July 2010.

14. Ramjan R, Calmy A, Vitoria M, Mills EJ, Hill A, Cooke G, et al. Systematic review and meta-analysis: Patient and programme impact of fixed-dose combination antiretroviral therapy. Tropical Medicine and International Health 2014; 19:501-513.

15. Luque-Fernandez MA, Van Cutsem G, Goemaere E, Hilderbrand K, Schomaker M, Mantangana N, et al. Effectiveness of patient adherence groups as a model of care for stable patients on antiretroviral therapy in Khayelitsha, Cape Town, South Africa. PLoS One 2013; 8:e56088. 
16. Cornell M, Grimsrud A, Fairall L, Fox MP, van Cutsem G, Giddy J, et al. Temporal changes in programme outcomes among adult patients initiating antiretroviral therapy across South Africa, 2002-2007. AIDS 2010; 24:2263-2270.

Copyright @ 2016 Wolters Kluwer Health, Inc. Unauthorized reproduction of this article is prohibited. 


\section{Figure legends}

Figure 1: Life expectancies from time of ART initiation (Model 1), compared to HIVnegative life expectancies

(a) Men starting ART at CD4 200+

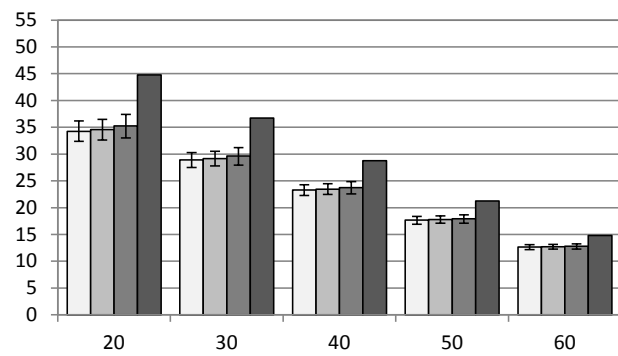

(c) Men starting ART at CD4 100-199

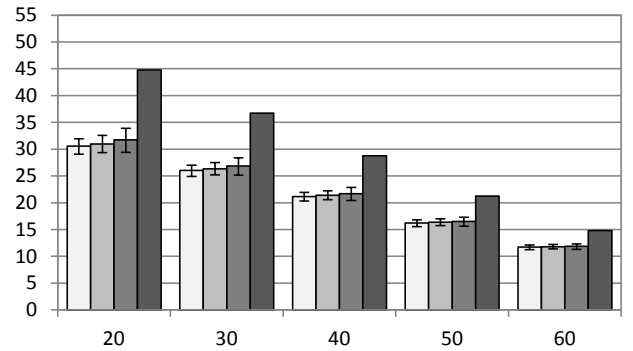

(e) Men starting ART at CD4 50-99

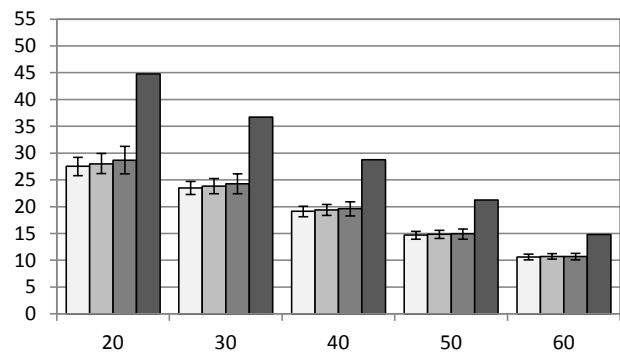

(g) Men starting ART at CD4 <50

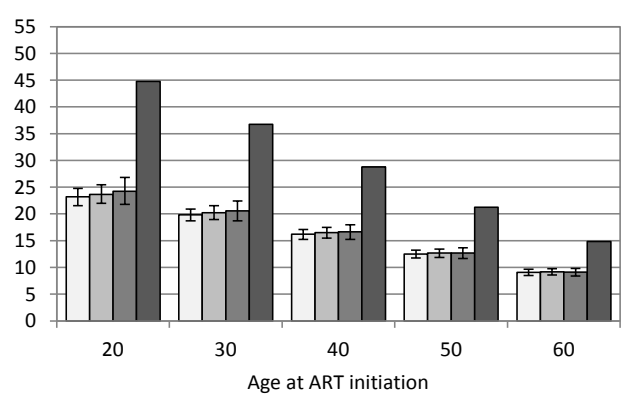

(b) Women starting ART at CD4 200+

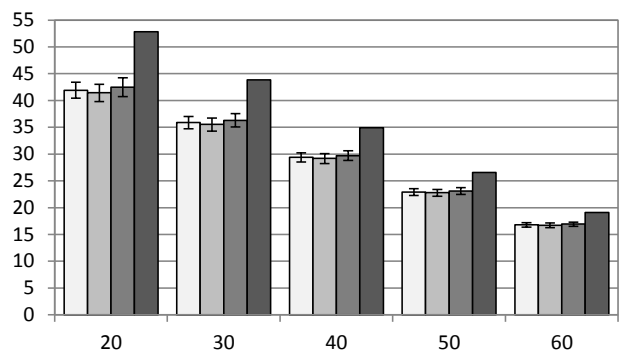

(d) Women starting ART at CD4 100-199

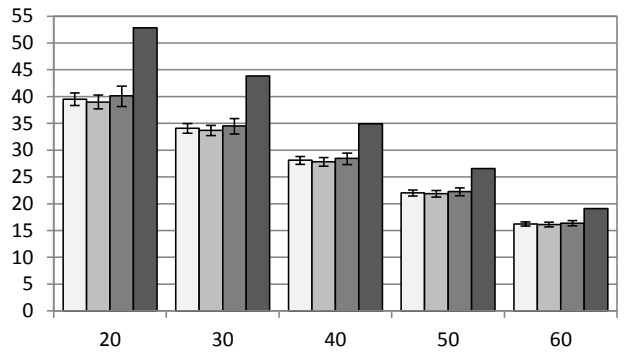

(f) Women starting ART at CD4 50-99

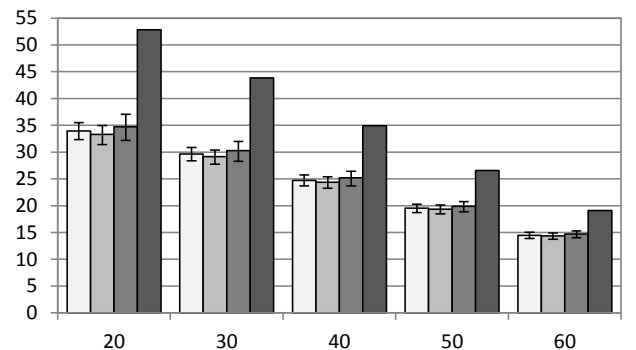

(h) Women starting ART at CD4 $<50$

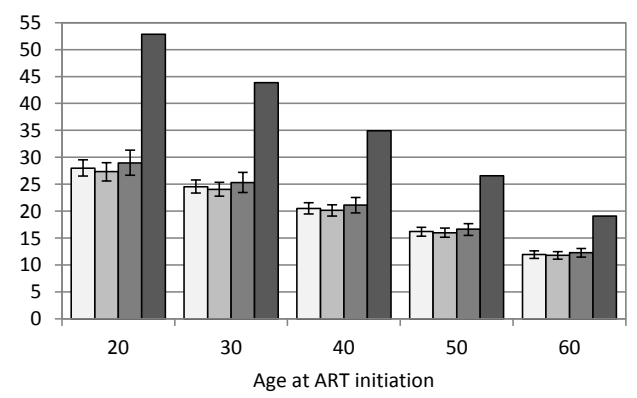

口Started ART in 2001-6 प $\quad$ Started ART in 2007-9

DStarted ART in 2010-4

DHIV negative 
Figure 2: Life expectancies of patients on ART by period of follow-up (Model 2, not controlling for baseline CD4 count and ART duration)

(a) Men

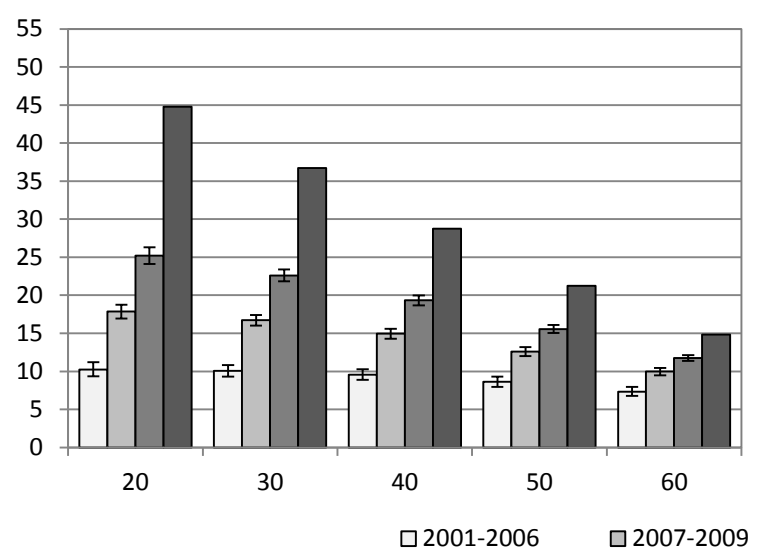

(b) Women

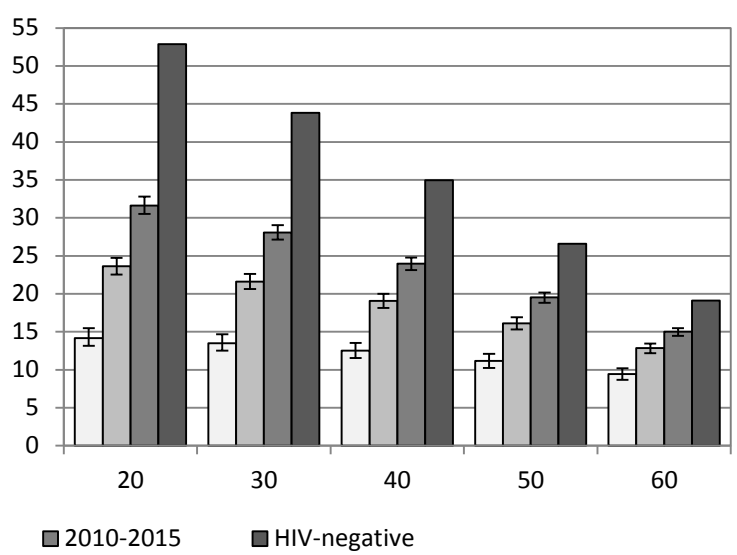

